\title{
Mechanism of microRNA-146a-mediated IL-6/STAT3 signaling in lumbar intervertebral disc degeneration
}

\author{
TAO ZHOU ${ }^{1}$, HAO LIN $^{2}$, ZIAO CHENG $^{2}$, CHAOCHAO JI $^{2}$, CHAO ZHANG $^{3}$ and JIWEI TIAN ${ }^{4}$ \\ ${ }^{1}$ Clinical Medical College, Shanghai General Hospital of Nanjing Medical University and Ma'anshan People's Hospital, \\ Anhui 243000; ${ }^{2}$ Medical University of Anhui, Hefei, Anhui 230000; ${ }^{3}$ Ma'anshan Clinical College of Medical \\ University of Anhui; ${ }^{4}$ Clinical Medical College, Shanghai General Hospital of Nanjing Medical University, \\ Hefei, Anhui 243000, P.R. China
}

Received April 4, 2017; Accepted May 30, 2017

DOI: $10.3892 /$ etm.2017.4611

\begin{abstract}
The aim of the study was to investigate the mechanism of microRNA (miR)-146a-mediated activation of interleukin-6/signal transducer and activator of transcription 3 (IL-6/STAT3) in lumbar intervertebral disc degeneration. To obtain intervertebral tissue, we recruited 5 patients with lumbar intervertebral disc herniation (experimental group) and 5 patients with lumbar burst fracture (control group). Nucleus pulposus tissue was extracted by surgery and cultured. miR-146a empty vector, mimic, and inhibitor were transfected into the two groups of cells for $24 \mathrm{~h}$ and the levels of IL-6, type II collagen (Col II), aggrecan, STAT3, matrix metalloproteinase (MMP)-3, and a disintegrin and metalloproteinase with thrombospondin type I motifs (ADAMTS) were detected. We found no differences in the levels of IL-6, Col II, aggrecan, STAT3, MMP-3, and ADAMTS before and after treatment in the control group. However, the levels of miR-146a, IL-6, STAT3, MMP-3, and ADAMTS were significantly elevated, whereas Col II and aggrecan levels were lower in the experimental group before treatment. The levels of IL-6, STAT3, MMP-3, and ADAMTS were elevated after treatment with miR-146a mimic when compared with the miR-146a empty vector in the experimental group, and Col II and aggrecan levels were decreased. However, the cells treated with miR-146a inhibitor had the opposite result. Thus, the IL-6/STAT3 signaling pathway can be mediated by a high expression of miR-146a to regulate the occurrence of lumbar intervertebral disc degeneration.
\end{abstract}

Correspondence to: Dr Jiwei Tian, Clinical Medical College, Shanghai General Hospital of Nanjing Medical University, 45 Hubei Road, Hefei, Anhui 243000, P.R. China

E-mail: hefang0688@163.com

Key words: microRNA-146a, interleukin-6/signal transducer and activator of transcription 3 signaling pathway, lumbar intervertebral disc degeneration, Col II, aggrecan, matrix metalloproteinase, a disintegrin and metalloproteinase with thrombospondin type I motifs

\section{Introduction}

Intervertebral disc degeneration (IDD) is the pathological basis of intervertebral disc protrusion, unstable spine, spinal canal stenosis, spinal cord, nerve root compression, and the major cause leading to low back pain (1). The reduction of type II collagen (Col II) and aggrecan contents are typical pathological changes of IDD and matrix metalloproteinase (MMP) is the most significant proteinase that can degrade the extracellular matrix (ECM) (2). A disintegrin-like and metalloproteinase with thrombospondin type I motifs (ADAMTS) also exerts important effects in degrading proteoglycans (3).

Previous findings showed that inflammatory reaction plays a significant role in the occurrence and development of IDD (4). The JAK2/STAT3 signaling pathway is key in central nervous system inflammation and immune response, and exerts an important role in synaptic plasticity, neurodegeneration, and memory formation (5). STAT3 is activated by the IL-6 cytokine and is regarded as a major factor mediating IL-6 function (6). Differential microRNA (miR) chip screening in degenerative nucleus pulposus indicates that the occurrence of IDD may be regulated by an abnormally high expression of miR-146a (7).

Based on this, we further analyzed the mechanism of miR-146a-mediated interleukin-6/signal transducer and activator of transcription 3 (IL-6/STAT3) activation in IDD. This study has the potential to identify a new target to improve our understanding of IDD and for therapeutic intervention.

\section{Materials and methods}

Patients and tissue collection. Five patients diagnosed with lumbar intervertebral disc herniation were selected as the experimental group and another five patients with lumbar burst fracture were enrolled as the control group. The experimental group had three males and two females, aged 50-70 years, with an average age of $57.5 \pm 8.9$ years. According to the position of disc damage, two cases affected lumbar spinal segments L2-3, two affected L4-5 and one affected L5-S1. The control group had three males and two females, aged 52-69 years, with an average age of $56.6 \pm 7.5$ years. The control group included two cases involving L3-4 and three cases affected L4-5. 
Written informed consent was obtained from patients. The study was approved by the Ethics Committee of Shanghai General Hospital of Nanjing Medical University.

Cell culture. The nucleus pulposus tissue of the lesion was removed by surgery, and cell culture and identification were performed. The tissue was digested with parenzyme and centrifuged at 2,000 $\mathrm{x}$ g for $5 \mathrm{~min}$. Subsequently, $0.2 \% \mathrm{Col}$ II was added and mixed uniformly for digestion within $4 \mathrm{~h}$. Dulbecco's modified Eagle's medium Nutrient Mixture F-12 (DMEM/F12) complete culture solution was added to stop the digestion. After filtration, the samples were centrifuged at $1,000 \mathrm{x}$ g for $5 \mathrm{~min}$ and DMEM/F12 complete culture solution was added. The cultures were re-suspended at a density of $1 \times 10^{5}$ cells $/ \mathrm{ml}$ and cultured at $37^{\circ} \mathrm{C}$, and $5 \% \mathrm{CO}_{2}$. The cultures were sub-cultured after the solution was changed the following day and cell attachment, growth, and morphological changes were observed under an inverted microscope (BX-42; Olympus, Tokyo, Japan). Second-generation cells were used for immunocytochemical staining to identify Col II expression in order to demonstrate the identity of the cultures as nucleus pulposus cells.

Research methods. Cell transfection was performed according to ribo FECTTMCP (Guangzhou RiboBio Co., Ltd., Guangzhou, China) transfection kit instructions. miR-146a empty vector, mimic and inhibitor were cultured with the two groups of cells for $24 \mathrm{~h}$ and transfection efficiency was detected. The levels of IL-6, Col II, and aggrecan were detected by ELISA, and the expression levels of STAT3, MMP-3, and ADAMTS were detected by western blotting.

Cell transfection. Cells were transferred onto 24-well plates at a density of $5 \times 10^{5}$ cells $/ \mathrm{ml}$. A total of $440 \mu \mathrm{l}$ DMEM/F12 (1:1) medium was added into each well and the transfection was performed when the cell volume reached $85 \%$. Then, $5 \mu \mathrm{l}$ $40 \mathrm{nM}$ miR-146a empty vector, miR-146a mimic, miR-146a mimic negative control, miR-146a inhibitor and miR-146a inhibitor negative control were added into $50 \mu 1$ FECTTMCP buffer solution, mixed well, and incubated at room temperature for $5 \mathrm{~min}$. FECTTMCP transfection agent $(5 \mu \mathrm{l})$ was added, mixed well, and incubated at room temperature for $15 \mathrm{~min}$. The 24-well plate was incubated at $37^{\circ} \mathrm{C}, 5 \% \mathrm{CO}_{2}$ for $24 \mathrm{~h}$. The cell transfection efficacy was detected by reverse transcription quantitative PCR.

ELISA method. IL-6, Col II and aggrecan agents were purchased from Beyotime Institute of Biotechnology (Jiangsu, China), and the cell extract was centrifuged at 3,000 $\mathrm{x}$ g for $20 \mathrm{~min}$ and detected by microplate reader. The average value was obtained in triplicate.

Western blotting. Radio-immunoprecipitation assay (RIPA) lysis buffer was added to the cells and the total protein was extracted. Coomassie brilliant blue was used for coarse quantitation and $\beta$-actin antibody was used for standardization. Total protein $(30 \mu \mathrm{g})$ was used for $8 \%$ sodium dodecyl sulfate-polyacrylamide gel electrophoresis (SDS-PAGE) separation. The proteins were transferred onto a polyvinylidene fluoride (PVDF) membrane, incubated overnight with mouse monoclonal STAT3 antibody (dilution, 1:500;

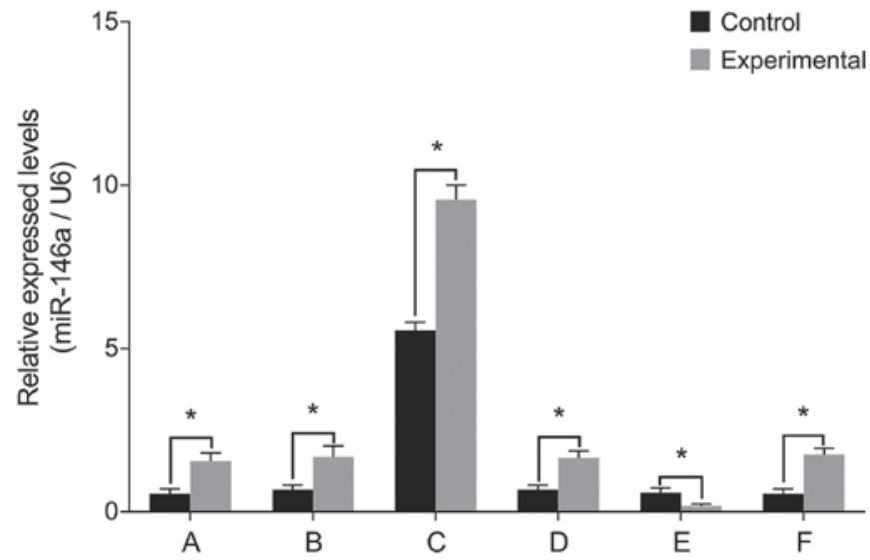

Figure 1. miR-146a mRNA levels after cell transfection. (A) miR-146a leve is higher in the experimental group than that in the control group before transfection. (B) miR-146a empty vector transfection. (C) miR-146a mimic transfection and miR-146a mRNA levels in the two groups of cells are increased 15-fold after treatment. (D) miR-146a mimic negative control transfection. (E) miR-146a inhibitor transfection and miR-146a mRNA levels in the two groups of cells are approximately decreased to $1 / 3$ of previous levels after treatment. (F) miR-146a inhibitor negative control transfection. No differences were identified before and after treatment. miR, microRNA. ${ }^{*} \mathrm{P}<0.05$

cat. no. AMAB90777), mouse monoclonal MMP-3 antibody (dilution, 1:500; cat. no. SAB530042) and mouse monoclonal ADAMTS antibody (dilution, 1:500; cat. no. WH0170691M1). Rabbit anti-mouse-HRP polyclonal secondary antibody (dilution, 1:2,000; cat. no. A9044MSDS) was incubated at room temperature for $4 \mathrm{~h}$. All the antibodies were purchased from Sigma (St. Louis, MO, USA). Phosphate-buffered saline (PBS) was used for washing and electrochemiluminescence (ECL) was used for signal development. The results were scanned and saved. Lab Works 4.5 gel imaging software (Invitrogen, Carlsbad, CA, USA) was used for semi-quantitative analysis.

Statistical methods. SPSS20.0 software (SPSS, Inc., Chicago, IL, USA) was used for statistical analysis. Measurement data were expressed as mean \pm standard deviation. Independent sample t-test was used for the intergroup comparison and one-way analysis of variance (ANOVA) was used for the comparison among multiple groups. Least significant difference (LSD) test was used for the paired comparisons and the paired t-test was used for a comparison before and after intervention in each group. Enumeration data were expressed as case or percentage, and the Chi-square test was used for the intergroup comparison. $\mathrm{P}<0.05$ indicated that the difference was statistically significant.

\section{Results}

miR-146a levels. To examine the role of miR-146a in the pathology of intervertebral discs, we cultured nucleus pulposus cells from patients with IDD and then manipulated miR-146a activity in these cells. The levels of miR-146a mRNA were significantly higher in the experimental group than those in the control group prior to transfection $(\mathrm{P}<0.05)$ (Fig. 1). Cell transfections confirmed that there were no differences between miR-146a mRNA levels before and after treatment with miR-146a empty vector, miR-146a mimic negative 


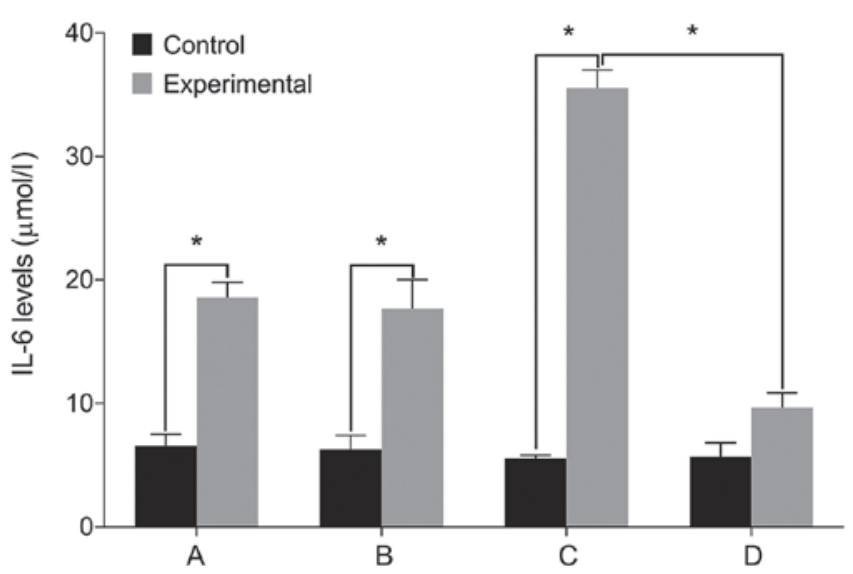

Figure 2. IL-6 levels by ELISA. (A) Before miR-146a transfection (B) miR-146a empty vector transfection. (C) miR-146a mimic transfection. (D) miR-146a inhibitor transfection. A comparison of IL-6 levels before and after treatment shows no difference in the control group. The cell IL-6 level is significantly higher in the experimental group than that in the control group before treatment. IL-6 level is increased after treatment with miR146a mimic in the experimental group, and is decreased after treatment with miR-146a inhibitor. IL-6, interleukin-6; miR, microRNA. "P<0.05.

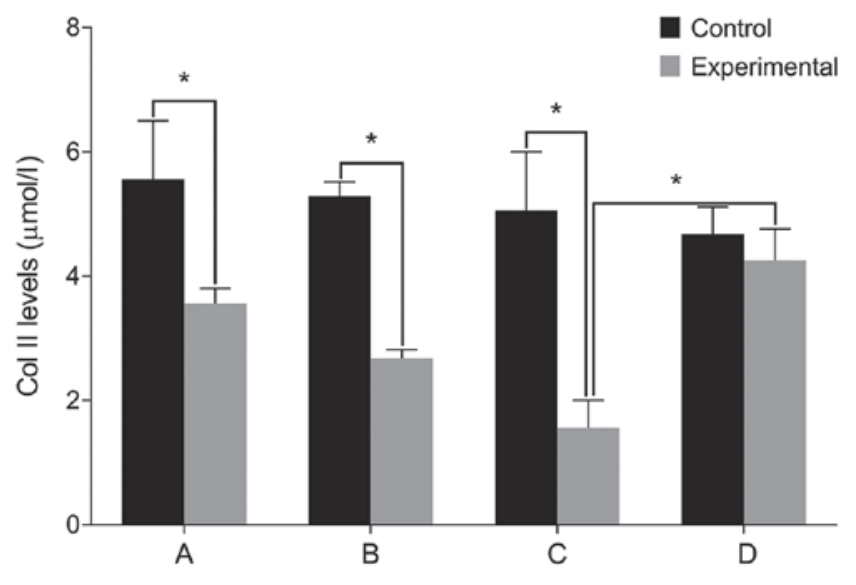

Figure 3. Col II levels by ELISA. (A) Before miR-146a transfection. (B) miR-146a empty vector transfection. (C) miR-146a mimic transfection. (D) miR-146a inhibitor transfection. There is no difference in Col II levels before and after treatment in the control group. The cell Col II level is significantly lower in the experimental group than that in the control group before treatment. Col II level is decreased after treatment with miR-146a mimic in the experimental group and is increased after treatment with miR-146a inhibitor. Col II, type II collagen; miR, microRNA. " $\mathrm{P}<0.05$.

control and miR-146a inhibitor negative control $(\mathrm{P}>0.05)$. miR-146a mRNA levels increased 15-fold after transfection with miR-146a mimic and decreased by $66 \%$ after transfection with miR-146a inhibitor.

IL-6, Col II and aggrecan levels. We then examined the expression of relevant markers in the cultured cells. We found no differences in the levels of IL-6, Col II and aggrecan before and after treatment in the control group $(\mathrm{P}>0.05)$. The level of IL-6 was significantly higher in the experimental group than that in the control group before treatment. The levels of $\mathrm{Col}$ II and aggrecan were lower in the experimental group compared with the control group $(\mathrm{P}<0.05)$. The level of IL-6 increased in the experimental group after treatment with

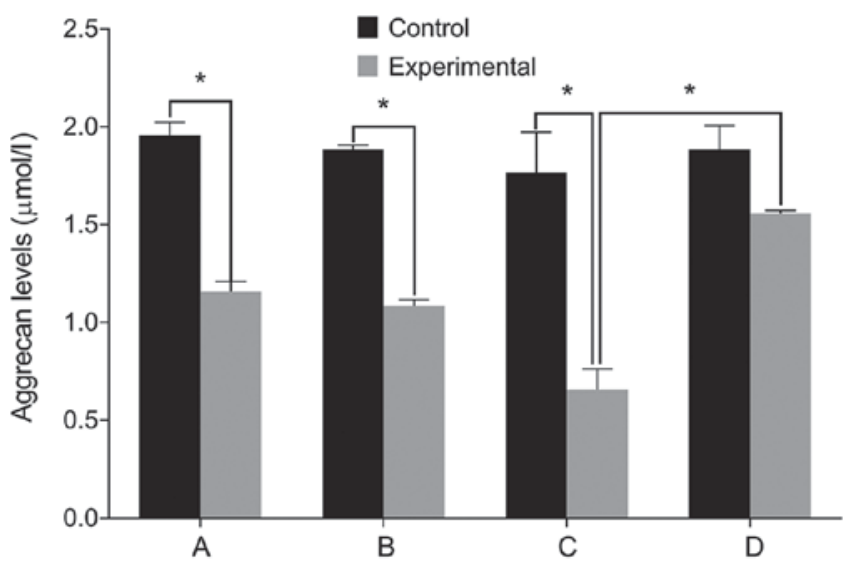

Figure 4. Aggrecan levels by ELISA. (A) Before miR-146a transfection. (B) miR-146a empty vector transfection. (C) miR-146a mimic transfection. (D) miR-146a inhibitor transfection. A comparison of aggrecan levels before and after treatment yielded no difference in the control group. The level of aggrecan is significantly lower in the experimental group than that in the control group before treatment. Aggrecan level is decreased after treatment with miR-146a mimic in the experimental group and is increased after treatment with miR-146a inhibitor. miR, microRNA. ${ }^{*} \mathrm{P}<0.05$.

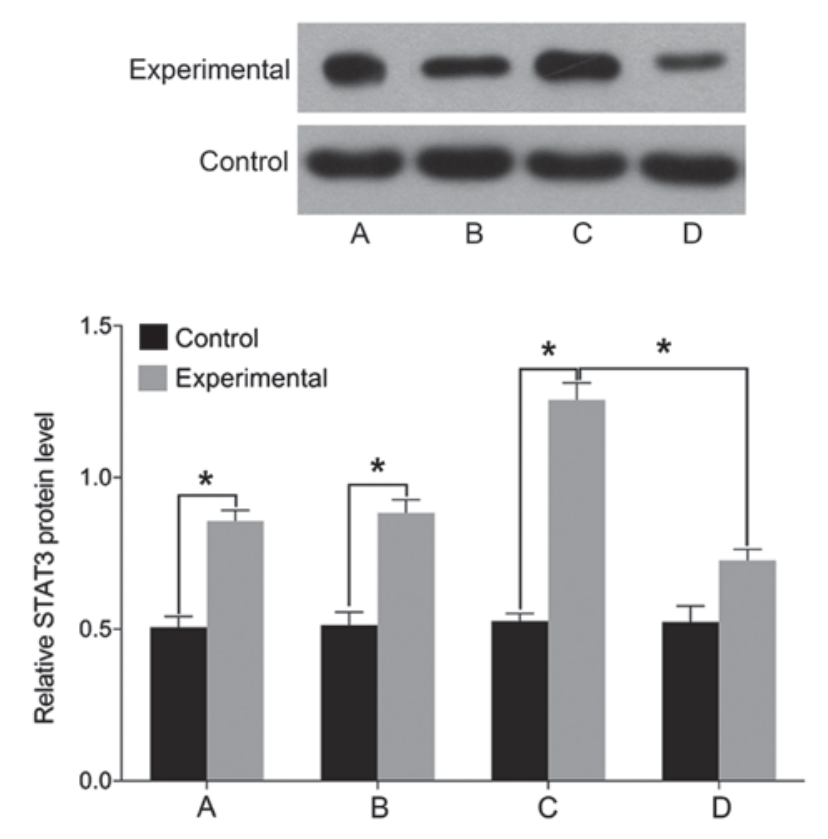

Figure 5. Detection of cell STAT3 levels by western blotting method. (A) Before miR-146a transfection. (B) miR-146a empty vector transfection. (C) miR-146a mimic transfection. (D) miR-146a inhibitor transfection. A comparison of STAT3 levels before and after treatment yielded no difference in the control group. The level of STAT3 is significantly higher in the experimental group than that in the control group before treatment. STAT3 level is increased after treatment with miR-146a mimic in the experimental group, and is decreased after treatment with miR-146a inhibitor. STAT3, signal transducer and activator of transcription 3 ; miR, microRNA. ${ }^{*} \mathrm{P}<0.05$.

miR-146a mimic when compared with the miR-146a empty vector. The levels of Col II and aggrecan levels decreased after treatment with miR-146a mimic $(\mathrm{P}<0.05)$. By contrast, the cells treated by miR-146a inhibitor had the opposite result $(\mathrm{P}<0.05)$ (Figs. 2-4).

STAT3, MMP-3 and ADAMTS levels. Finally, we examined the expression of markers associated with intervertebral disc 

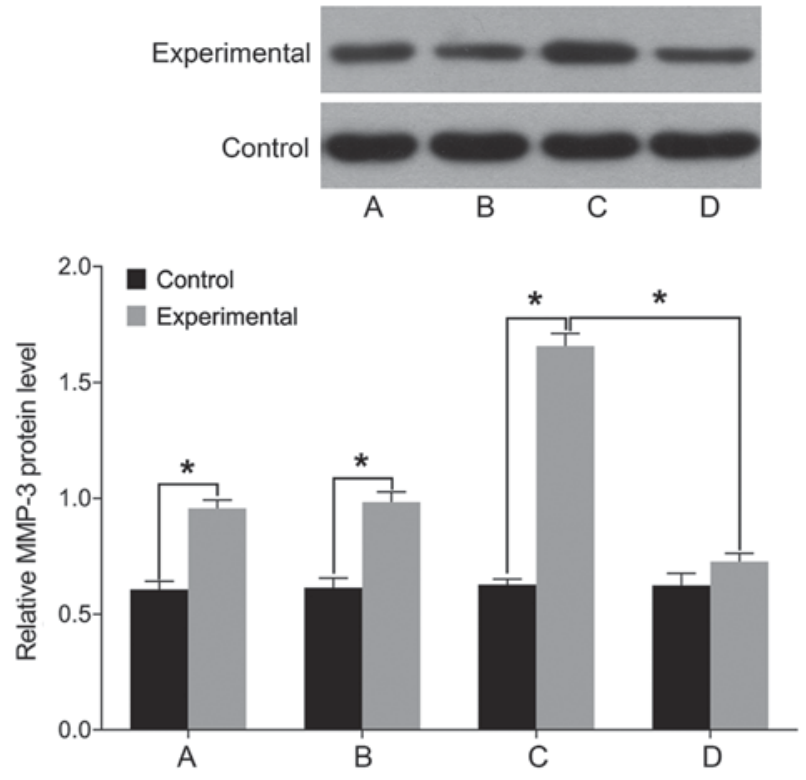

Figure 6. Detection of cell MMP-3 levels using western blot analysis. (A) Before miR-146a transfection. (B) miR-146a empty vector transfection. (C) miR-146a mimic transfection. (D) miR-146a inhibitor transfection. A comparison of MMP-3 levels before and after treatment yielded no difference in the control group. The cell MMP-3 level is significantly higher in the experimental group than that in the control group before treatment. MMP-3 level is increased after treatment with miR-146a mimic in the experimental group, and is decreased after treatment with miR-146a inhibitor. MMP-3, matrix metalloproteinase; miR, microRNA. ${ }^{*} \mathrm{P}<0.05$.

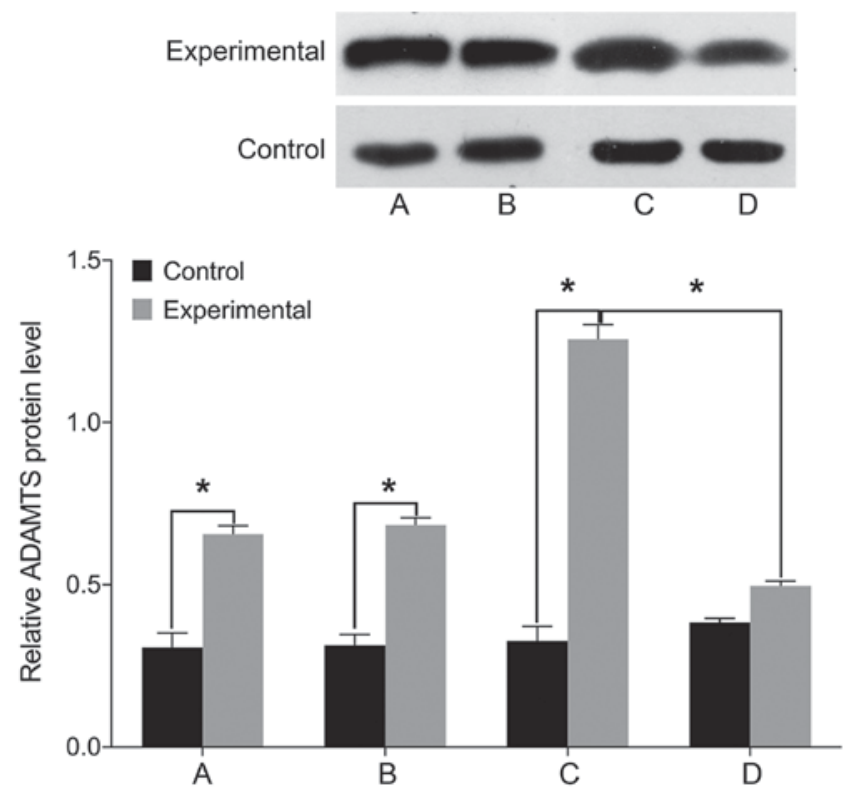

Figure 7. ADAMTS levels by western blotting. (A) Before miR-146a transfection. (B) miR-146a empty vector transfection. (C) miR-146a mimic transfection. (D) miR-146a inhibitor transfection. A comparison of ADAMTS levels before and after treatment yielded no differences in the control group. The cell ADAMTS level is significantly higher in the experimental group than that in the control group before treatment. ADAMTS level is increased after treatment with miR-146a mimic in the experimental group, and is decreased after treatment with miR-146a inhibitor. ADAMTS, a disintegrin and metalloproteinase with thrombospondin type I motifs; miR, microRNA." $\mathrm{P}<0.05$.

pathology. We found no differences in the levels of STAT3, MMP-3, and ADAMTS before and after treatment in the control group ( $\mathrm{P}>0.05)$. The levels of cell STAT3, MMP-3, and ADAMTS were significantly higher in the experimental group than those in the control group before treatment $(\mathrm{P}<0.05)$. In the experimental group, the levels of STAT3, MMP-3, and ADAMTS were elevated following treatment with miR-146a mimic and decreased after treatment with miR-146a inhibitor $(\mathrm{P}<0.05)$ (Figs. 5-7).

\section{Discussion}

Results of the present study revealed that treatment of control cells with miR-146a had no effect on the levels of the pathogenic markers IL-6, Col II, aggrecan, STAT3, MMP-3 and ADAMTS, suggesting that miR-146a is not the initiating factor in the degeneration of nucleus pulposus. The levels of miR-146a, IL-6, STAT3, MMP-3 and ADAMTS were significantly higher in the experimental group, whereas Col II and aggrecan were lower in the experimental group. The abnormally high expression of miR-146a was positively correlated with the Pfirrmann classification of degeneration (8). The study of $\mathrm{Gu}$ et al indicated that miR-146a expression is associated with intervertebral disc IL-1-mediated inflammatory reaction (9).

Cell transfection experiments suggested that IL-6/STAT3 signaling pathway can be activated by a high expression of $\mathrm{miR}-146 \mathrm{a}$ to promote lumbar intervertebral disc degeneration. Bioinformatics analysis revealed that IL-6/STAT3 may be a direct target of miR-146a (10). An IDD rat model demonstrated that the inflammatory reaction with elevated IL-6 and TNF- $\alpha$ and prolonged duration was highly significant (11). Recombinant IL-6 injected in the rat lumbar dorsal root ganglion can lead to hyperalgesia, but the trigger pain can be significantly reduced by IL- 6 inhibitors. The mechanical hyperalgesia can be alleviated by silencing the $I L-6$ gene (12). Additionally, anti-inflammatory cytokines such as IL-10 show a significant analgesic effect (13). Burke et al also indicated that the high level of inflammatory molecules secreted by intervertebral disc tissue is an important pathogenic factor leading to low back pain (14). JAKs can be activated through IL-6 binding with its receptor, activating STAT3 phosphorylation, which enables its nuclear translocation where it combines with the promoters of its target genes. Inflammation is an important factor that can promote tumor formation and malignant lesions (15). Efficient blocking of IL-6/STAT3 signaling pathway can be achieved with multiple small molecule inhibitors, such as STA-21 and STX-0119, which may be beneficial in the treatment of IDD (16). Apoptosis and the increase of proteases are major reasons leading to the reduction of ECM component in the intervertebral disc. MMPs and ADAMTS are $\mathrm{Zn}^{2+}$-dependent proteases with broad substrate specificity that can be activated by multiple inflammatory factors, including as IL-6 and TNF- $\alpha(17,18)$.

In summary, this study revealed that IDD is associated with high levels of miR-146a, inflammatory reaction due to IL-6 and activation of the STAT3 signaling pathway, thereby affecting the expression of $\mathrm{Col}$ II and aggrecan in the nucleus pulposus tissue. Future studies are to investigate whether IDD can be reversed by the single or combined effects of targeting miR-146a, IL-6, or STAT3, which will provide an important reference for the clinical intervention in IDD. 


\section{References}

1. Ma T, Guo CJ, Zhao X, Wu L, Sun SX and Jin QH: The effect of curcumin on NF- $\kappa \mathrm{B}$ expression in rat with lumbar intervertebral disc degeneration. Eur Rev Med Pharmacol Sci 19: 1305-1314, 2015.

2. Wang X, Wang H, Yang H, Li J, Cai Q, Shapiro IM and Risbud MV: Tumor necrosis factor- $\alpha$ - and interleukin-1 $\beta$ dependent matrix metalloproteinase-3 expression in nucleus pulposus cells requires cooperative signaling via syndecan 4 and mitogen-activated protein kinase-NF- $\kappa \mathrm{B}$ axis: Implications in inflammatory disc disease. Am J Pathol 184: 2560-2572, 2014.

3. Sun Z, Yin Z, Liu C, Liang H, Jiang $M$ and Tian J: IL-1 $\beta$ promotes ADAMTS enzyme-mediated aggrecan degradation through NF- $\kappa$ B in human intervertebral disc. J Orthop Surg 10: $159,2015$.

4. Liu C, Fei HD, Sun ZY and Tian JW: Bioinformatic analysis of the microarray gene expression profile in degenerative intervertebral disc cells exposed to TNF- $\alpha$. Eur Rev Med Pharmacol Sci 19: 3332-3339, 2015

5. Zhang SY, Zhao QF, Fang NN and Yu JG: Betulin inhibits pro-inflammatory cytokines expression through activation STAT3 signaling pathway in human cardiac cells. Eur Rev Med Pharmacol Sci 19: 455-460, 2015.

6. Lu X, Luo F, Liu Y, Zhang A, Li J, Wang B, Xu W, Shi L, Liu X, Lu L, et al: The IL-6/STAT3 pathway via miR-21 is involved in the neoplastic and metastatic properties of arsenite-transformed human keratinocytes. Toxicol Lett 237: 191-199, 2015.

7. Ye EA and Steinle JJ: miR-146a attenuates inflammatory pathways mediated by TLR4/NF- $\mathrm{BB}$ and $\mathrm{TNF} \alpha$ to protect primary human retinal microvascular endothelial cells grown in high glucose. Mediators Inflamm 2016: 3958453, 2016.

8. Zhao B, Yu Q, Li H, Guo X and He X: Characterization of microRNA expression profiles in patients with intervertebral disc degeneration. Int J Mol Med 33: 43-50, 2014.

9. Gu SX, Li X, Hamilton JL, Chee A, Kc R, Chen D, An HS Kim JS, Oh CD, Ma YZ, et al: MicroRNA-146a reduces IL-1 dependent inflammatory responses in the intervertebral disc. Gene 555: 80-87, 2015.
10. Hu P, Feng B, Wang G, Ning B and Jia T: Microarray based analysis of gene regulation by microRNA in intervertebral disc degeneration. Mol Med Rep 12: 4925-4930, 2015.

11. Risbud MV and Shapiro IM: Role of cytokines in intervertebral disc degeneration: Pain and disc content. Nat Rev Rheumatol 10: 44-56, 2014.

12. Deng X, Zhao F, Kang B and Zhang X: Elevated interleukin-6 expression levels are associated with intervertebral disc degeneration. Exp Ther Med 11: 1425-1432, 2016

13. Molinos M, Almeida CR, Caldeira J, Cunha C, Gonçalves RM and Barbosa MA: Inflammation in intervertebral disc degeneration and regeneration. J R Soc Interface 12: 20141191, 2015.

14. Burke JG, Watson RWG, McCormack D, Dowling FE, Walsh MG and Fitzpatrick JM: Intervertebral discs which cause low back pain secrete high levels of proinflammatory mediators. J Bone Joint Surg Br 84: 196-201, 2002.

15. Johnson ZI, Schoepflin ZR, Choi H, Shapiro IM and Risbud MV: Disc in flames: Roles of TNF- $\alpha$ and IL-1 $\beta$ in intervertebral disc degeneration. Eur Cell Mater 30: 104-116, discussion 116-117, 2015.

16. Miao D and Zhang L: Leptin modulates the expression of catabolic genes in rat nucleus pulposus cells through the mitogen-activated protein kinase and Janus kinase 2 /signal transducer and activator of transcription 3 pathways. Mol Med Rep 12: 1761-1768, 2015.

17. Wang XH, Zhu L, Hong X, Wang YT, Wang F, Bao JP, Xie XH, Liu L and Wu XT: Resveratrol attenuated TNF- $\alpha$-induced MMP-3 expression in human nucleus pulposus cells by activating autophagy via AMPK/SIRT1 signaling pathway. Exp Biol Med (Maywood) 241: 848-853, 2016.

18. Tian Y, Yuan W, Fujita N, Wang J, Wang H, Shapiro IM and Risbud MV: Inflammatory cytokines associated with degenerative disc disease control aggrecanase-1 (ADAMTS-4) expression in nucleus pulposus cells through MAPK and NF- $\mathrm{kB}$. Am J Pathol 182: 2310-2321, 2013 